Cahiers d'études italiennes

$7 \mid 2008$

NOVECENTO... E DINTORNI

Images littéraires de la société contemporaine (3)

\title{
Luoghi sub specie alteritatis. Giovanni Testori
}

\section{Silvia Giuliani}

\section{OpenEdition}

\section{Journals}

Edizione digitale

URL: http://journals.openedition.org/cei/945

DOI: $10.4000 /$ cei. 945

ISSN: 2260-779X

\section{Editore}

UGA Éditions/Université Grenoble Alpes

\section{Edizione cartacea}

Data di pubblicazione: 15 maggio 2008

Paginazione: 373-383

ISBN: 978-2-84310-121-2

ISSN: $1770-9571$

Notizia bibliografica digitale

Silvia Giuliani, «Luoghi sub specie alteritatis. Giovanni Testori », Cahiers d'études italiennes [Online], 7 | 2008, Messo online il 15 novembre 2009, consultato il 24 avril 2019. URL : http:// journals.openedition.org/cei/945; DOI : 10.4000/cei.945 


\title{
LUOGHI SUB SPECIE ALTERITATIS GIOVANNI TESTORI
}

\author{
Silvia Giuliani \\ Université de Pise, École pratique des Hautes études-Paris 4
}

L'impressione destata dalla Trilogia ${ }^{1 *}$ di Giovanni Testori in coloro che hanno potuto assistere negli anni Settanta alla messa in scena di uno dei tre drammi è quella di un teatro "differente» dalla prassi scenica e dalle convenzioni teatrali contemporanee ${ }^{2}$ : diverso suonava innanzitutto il linguaggio nel suo improbabile impasto di dialetto lombardo, latinismi e francesismi, diversi i personaggi e le loro battaglie rispetto ai modelli tragici codificati dalla tradizione, diversi i costumi e le scene quasi presi in prestito da un cabaret d'accatto, diverso il luogo fisico delle rappresentazioni, il Salone Pier Lombardo di Milano ${ }^{3}$, nonché la persona dell'attore, Franco Parenti, in funzione del quale i drammi erano stati concepiti.

Anche per chi, come in questo caso, elimina gli aspetti inerenti la messa in scena per basarsi su un'analisi esclusivamente letteraria dei testi in questione, che spesso per la loro struttura a monologo presentano forti caratteri di narratività, restano comunque in gioco diversi livelli in cui si articola il motivo dell'alterità.

Uno per tutti, quello al quale tutti gli altri risultano inesorabilmente legati, è il piano della costruzione del personaggio, da cui questa disamina prenderà le mosse.

La posizione di diversità conflittuale di cui gode statutariamente l'eroe tragico rispetto ad una collettività è amplificata e portata al massimo grado, nei personaggi della Trilogia, da una sovrapposizione tra due momenti temporali diversi, quello del presente della rappresentazione e quello mitico in cui la vicenda tragica è ambientata, ma soprattutto da un conseguente cortocircuito fra il personaggio di primo grado, derivato dalla tradizione letteraria, e il personaggio-attore di secondo grado, lo Scarrozzante, carico del suo personale dramma che va a confondersi con la traccia drammatica di base. Questi attori girovaghi della Brianza degli

* Notes p. 382. 
anni Trenta chiamati "scarrozzanti» facevano delle campagne e delle piazze di paese il teatro delle loro istintive messe in scena, rifacimenti abborraciati di pièces in cui spesso finivano per convogliare la loro personale esperienza, le loro proteste e rivendicazioni, creando una sovrapposizione di teatro e vita che non poteva non colpire profondamente l'immaginazione di Testori.

Ed è da questo momento che il mondo degli scarrozzanti, ovvero, secondo le parole dell'autore stesso, "dei reietti, dei diversi, dei fuori norma, dei non accettati dai partiti e dalle chiese», di quelli «per cui la vita è fatale solitudine, autodistruzione, girare, andare ${ }^{4}$ ", entra a far parte della scrittura drammaturgica testoriana, determinando con la sua invadente presenza uno slittamento nella vicenda tragica presupposta per far posto ad una nuova, ulteriore diversità.

Il principe Amleto si presenta così come un guitto di provincia che, dando il via metateatralmente all'azione tragica, presenta la ditta di cui fa parte e stabilisce come ambientazione un'imprecisata località che potrebbe essere «Elzinore», o Camerlata, o Lomazzo, o la «Mediolanensis urbiz ", in un tempo volutamente lasciato in sospeso che rinvia tanto ad un fosco Medioevo o al Settecento, quanto ai nostri giorni.

Il giovane dimentica gli attributi di principe intellettuale per esprimere la sue inquietudini di zingaro insofferente ai ruoli e al potere, tormentato da angosciose domande sul senso della vita e della nascita, interiormente scisso tra "Am» e «bleto ", senza riuscire a capire come potrà un giorno ricongiungere i due brani di sé per trovare nuovo significato.

Tempo prima, in preda ad una profonda crisi esistenziale, Ambleto racconta di essere stato salvato da morte sicura dal «Franzese ", "angioro incarnato » che lo aveva saputo condurre sulla strada dell'amore universale e oblativo, l'unico sentimento per cui la vita meriterebbe di essere vissuta e che potrebbe riscattare l'umanità dal suo stato di degrado. Per quanto però ci si guardi intorno, l'amore non sembra aver lasciato alcuna traccia dopo essersi sottratto ad una terra fatta come una "piramida» nella quale possono avere ormai asilo solo le scalate ai più alti gradi sociali, alle onorificenze, alla ricchezza e al potere. Tale è il regno di Arlungo, lo zio del protagonista, che si è impadronito del potere dopo la morte del fratello e ne sta per sposare la moglie: in siffatto regno non possono trovar spazio gli insegnamenti del Franzese, il novello Orazio scacciato dalla famiglia di origine perchè "diverso ${ }^{5}$ » e invitato con scopi polizieschi dai sovrani, ma presto bollato da questi come un "narchico, e pergiunta 'vuno de quei' narchi che credono anca in del Cristo! [...] Bello, amadore de vuomini e de donne, zobillante e cont el Cristo, qui, in zul cuore!» (p. 1173). 
Ecco dunque che Ambleto, da bravo scarrozzante e in una sorta di doppio rispetto al Franzese, cercherà di realizzare nel suo regno quella promessa di amore che la colpa degli uomini ha disatteso, di ristabilire un ordine basato sull'eguaglianza e la comunione fra gli uomini, senza violenza, repressione, prevaricazione, dove non ci siano diversi né emarginati.

L'apparizione dello spettro del padre in un altro dei momenti di più profonda disperazione di Ambleto non aiuta a trovare risposta alcuna ai suoi interrogativi angosciosi, ma l'uomo, dopo aver rivelato la verità circa la sua morte, o meglio il suo assassinio tramite una "formaggella» avvelenata, e dopo essersi rivolto ad Ambleto con una chiara ingiunzione ( $\mathrm{Ma}$ te non facere neintus conta el regno... Salva la statutazzione; salva la corona; salva la granda, soveràna piramida dell'ordeno e del potere; salvala... », p. 1185), sparisce nel nulla.

Questo ordine non è per Ambleto che la goccia che fa traboccare il vaso: d'ora in avanti ogni suo sforzo sarà ridotto alla distruzione della «piramida» e della "statutazzione», "a comenzare dall'Unico e Unichissimo che ce sta sù, in la cima!» (p. 1185), ma ogni sforzo tendente ad una nuova vita si conclude pessimisticamente in una sequela di morti che alla fine coinvolgerà il protagonista stesso, nel nulla e nella distruzione totale senza un Laerte o un Fortebraccio in grado di ristabilire, seppur momentaneamente, gli equilibri sconvolti. I tempi non sono ancora maturi perché si possa pensare ad un ordine capace di integrare una diversità che dà "scandalo ${ }^{6}$; l'unica alternativa è ancora il nulla.

Mentre l'apparizione dello spettro nella tragedia shakespeariana contribuiva a creare una piattaforma di consentaneità tra padre e figlio sulla quale innestare la comune vendetta e giungere a ristabilire l'ordinamento del sistema sociale che era stato scardinato dalla morte violenta del legittimo sovrano, nell'analoga scena in Testori emerge invece la volontà di un figlio di opporsi al padre, o meglio ai padri ${ }^{7}$ e al regime da loro colpevolmente fondato e perpetrato, in nome di valori diversi su cui basare la vita sociale e politica. Quando anche una madre, l'istanza femminile, l'unico appiglio per realizzare un nuovo mondo fondato sull'amore, si compromette con il potere onnipresente e onnipervasivo, non merita pietà: a differenza del modello elisabettiano sarà Amleto ad uccidere Gertruda prima di togliersi la vita.

Allo stesso risultato nullificante pervengono coloro che, invece di distruggere in nome di un'alterità, cercano di integrarsi e di inerpicarsi su per la "piramida » : questa è la sorte del Macbetto-scarrozzante di Testori.

Il principio del male che si impadronisce del Macbeth shakespeariano tramite il sortilegio delle streghe risulta in questo caso interno al protago- 
nista stesso e momentaneamente oggettivato in funzione drammatica tramite la figura fantasmatica di una strega generata da una defecazione indotta da pillole di fuca consigliate a Macbetto dalla Ledi. Le allettanti profezie acquistano però la consistenza di una sequela di assassinii solo grazie all'intervento prepotente e persuasivo della donna, descritta fin dall'inizio dallo stesso Macbetto come dotata di tratti fortemente virili, la principale depositaria del "cazzo del potero e del dominamento" (p. 1245), una sorta di Clitennestra dal «cuore capace di maschi pensieri $^{8}$ ", la cui colpevolezza è esaltata fino ai limiti dell'attacco misogino nella versione testoriana. Quello che non si perdona alla donna è ancora una volta l'aver annullato la peculiarità del principio femminile, principio dell'amore e della condivisione, nel "principio "maschile" della prevaricazione e del potere" ".

Le perplessità iniziali di Macbetto sfociano in vero e proprio senso di colpa nel momento del sacrificio dell'amico Banco, culmine dell'azione tragica e punto di svolta verso il rinnegamento di quanto è stato finora compiuto. È proprio il tradimento dell'amore che ha innescato questa tragica scalata e dal momento di chiedersi: "amare, qui, in'sta terra, o anca solamente non ancidere,/se poderà proprio mai più?» (p. 1292), al momento di scagliarsi con ferocia omicida nei confronti della donna, passa davvero un breve lasso di tempo nella mente di Macbetto. Mentre la Ledi, pugnalata a morte, canta un inno al potere che d'ora in avanti si chiamerà indicativamente "potera ", sferra anche l'ultimo distruttivo assalto nei confronti di Macbetto, avverando la profezia secondo la quale sarebbe stato ucciso da un individuo né femmina, né maschio.

Ancora una volta ogni alternativa è negata in un meccanismo che schiaccia coloro che si avvicinano. L'ossessione del potere (ormai sinonimo dell'ineliminabile male di vivere) ha ucciso i due sanguinari protagonisti senza possibilità di ritrattazione. Macbetto può solo constatare che senza amore «la vita non è vita. È solo un vurlo,/un ciurlo;/o forse un uè uè... / E poi? Nigora, demenza,/fabbreca de morte, sfantascienza... [...] Non c'è speranza più " (p. 1319-1320) 0; nell'indeterminato regno alpestre di Macbetto come nella valle della Brianza raccontata dallo scarrozzante.

Anche l'ultimo dramma della Trilogia, Edipus, è caratterizzato da un'ambientazione vaga e una collocazione temporale che può rimandare ora all'antica Tebe, ora alla contemporaneità, tanto più in quanto più marcatamente che negli altri drammi questa è la tragedia di Edipo ma, in non diversa misura, anche dello Scarrozzante, solo sulla scena a interpretare tutti i ruoli e a raccontare, in un intreccio inestricabile fra le due 
trame, di essere stato abbandonato sia dalla prima attrice, sua ex amante, che ha cercato una sistemazione borghese nel matrimonio con un mobiliere di Meda, sia dal giovane attore che ha preferito una compagnia di cabarrettisti. Tra cianfrusaglie e chincaglierie, rabbia e ricordi elegiaci, si erge l'ultimo depositario di un modo diverso di far teatro.

Fin dalla prima scena, in cui lo scarrozzante si affretta ad indossare gli abiti di Laio, emerge con chiarezza la problematica fondamentale del dramma qui maggiormente caricata rispetto ai drammi precedenti di chiare connotazioni politiche e sociali e di riferimenti polemici alla scottante attualità del «compromesso storico » fra Marx e Vangelo: il totalitarismo repressivo dei padri contro la trasgressione edipica portatrice di valori alternativi e individuali.

Una lampante descrizione del primo versante del binomio è fornita da Laio stesso che presenta la sua persona e il suo regno nel momento di accingersi a processare tre traditori dell'ordine costituito:

Re [...] e, nell'istessissimo tempo, Pontifex maximum, maximum et unichissimum, della qui insiemata e coincarnata Giesa, tebanica o tebaica anca lei; la qual Giesa et el qual Regno [...] se son reuniti, imbracciati, inlettati, fusi, perfusi, inchiavati e incoitati dedentro el grembo maternissimo della storia, vegnendo a formare un'unità mai prima de'desso vista e cognossuta; un'unità impastata int una solissima tessitazione de volontà, de ardori, de amori, de’bligazzioni, de statuti, de camare senatoriali e deputatoriali, de parrocchie, de ordini, de conventualità [...]. Unità la quale est mondaniga et, insieme, devina; politiga et, insieme, estraterrica; socialiga et insieme clericaliga; et anchis, in del suo più alto segnificarsi, metafisica, patafisiga et metabolica. (p. 1328-1329)

Un regno tale deve essere mantenuto costantemente puro e libero da ogni presenza "anti »: il primo a dare il buon esempio è stato Laio stesso sacrificando il proprio figlio che i vaticini avevano bollato come futuro disgregatore dello stato. Si procede dunque ai sommari processi e alle esecuzioni, la cui descrizione è complementare a quella appena riportata ai fini della delineazione dello stato. La prima vittima è accusata di crimini contro la chiesa, avendo calpestato un'ostia e gridato, brandendo i genitali, che Cristo è una "vaccata» inventata dai preti e dai "socialighi» (p. 1333); il secondo uomo è stato sorpreso a pulirsi il sedere con la bandiera "partitiga, giesastiga et operariga» (p. 1334), motivo più che sufficiente per meritare la ghigliottina; il terzo, scandalo degli scandali, è un bellissimo giovane che invece di sfruttare le sue doti facendo il ballerino si è ridotto allo stato di omosessuale e, come se non bastasse, «Scandalistego e infanticidigo dell'anima e della carne altruiche» (p. 1335). «El contralex, el contrasocietas, el contranatura» (p. 1335) è stato infatti sorpreso a commettere atti orali con un quindicenne e ad avanzare anche la pretesa 
che le guardie gli lasciassero finire l'opera intrapresa prima di condurlo in prigione. Laio ordina che gli venga tagliata la gola.

Lo Scarrozzante prende alternativamente le vesti di Giocasta e quelle di Laio inscenando un dialogo in cui emerge una donna in preda ai sensi di colpa per aver permesso al marito di sacrificare il neonato in nome dello stato "tebanico" e speranzosa che questi da qualche parte possa essere ancora vivo.

A confermare la madre nelle sue aspettative fa la sua apparizione l'Edipo-scarrozzante che senza troppi ambagi cerca subito il confonto diretto col padre. Veniamo presto a sapere che il neonato è stato tratto in salvo dalle belve del Citerone e cresciuto da un pastore e che, fattosi adulto, è stato informato delle sue vicissitudini e formato al suo credo da un dio diverso da quelli venerati nello stato tebano: Dioniso. Contro l'ordinamento apollineo già macchiato di socratismo e il principio di individuazione si erge Dioniso che, in quanto principio dell'indistinto e dell'unità originaria, secondo la tesi nietzschiana che l'autore fa propria, si pone come fondamento culturale della rivoluzione in atto. L'inno con cui Edipo presenta ed esalta la propria divinità in quanto canto della diversità fa da perfetto contraltare all'elogio fatto da Laio all'unità e « totalità » dello stato:

existe no domà la sfingia a fa i oraculi! Existe anca el Dioniso! Lui, el dio luciferico, el follo, el'briaco, el baccante, l'ispiradore dei poeti, dei lunateghi, dei malinconighi, dei diseredati, dei deversifigati, dei reietti, dei degetti, dei oscurati de mente, dei maledetti dal Cristo e dal Marxo [...]. El dio che protegge, envoca e dimanda no l'ordino, imbensì el disordino : no la'bedienza imbensì la revolta, la rebelliona, la sovversività, la ruina e la destruzion d'ogni et qualsiasi legge, la'narchia completa e universaliga [...] lui, el dioniso m'ha revelato su tutto! Tutto, pater! El tuo piano, el tuo disegno, el tuo'sassinio! (p. 1351-1352)

Da Dioniso ha saputo che Laio è il suo peggior nemico e che deve vendicarsi contro di lui, in nome di tutti coloro che rischiano di essere annullati e rimossi dalla prevaricante totalità dello stato paterno, e allo scopo di non consentire che in nome di un tale leviatano un giorno qualcuno possa imporre: «basta'namoramenti, basta feste, basta gioiezze, basta figazioni, basta inculazioni, basta felicità et felicitazioni [...] basta occhi popille e bocche de fiori, basta at ogni et qualunque libertà » (p. 1352-1353).

Non resta che procedere senza esitazione alcuna all'evirazione e sodomizzazione del padre.

Il momento cardine dell'incontro con Giocasta non tarda ad arrivare e sempre con la stessa decisione e la stessa violenza viene perpetrato l'incesto, e inferto in tal modo anche l'ultimo supremo colpo all'autorità paterna $^{10}$. 
Contrariamente alle aspettative, l'impatto dell'incesto su Giocasta sembra aver sortito l'effetto di risvegliarla improvvisamente dopo anni di letargo e di farla sentire per la prima volta viva. Ai dubbi se sia giusto uccidersi per essersi macchiata di una tale colpa davanti alle leggi del «decalogo Unitarigo e Testamentarigo " e "per offerire a tutti gli unifigati un àltero sanguinanto et ciarissimo exempio de come tutto et omnia se sacrifighi all'Unetà, anca la vita!» (p. 1367), la donna si fa afferrare del tutto dalle sensazioni paradisiache del suo risveglio a nuova luce e in uno slancio vitalistico, rinnegate le "patrie, leggi, leggiferazioni, corone ", chiede a «Ledipus» di portarla con sé a Colono, nel nuovo stato di natura, nel mitico regno matriarcale dell'amore.

L'unità mistica dei due, secondo la quale Edipo è diventato «creatoro » oltre che creato, è però improvvisamente dilaniata da una raffica di mitra dalle quinte che uccide madre e figlio. Non sono però dei perdenti perché hanno contribuito ad aprire una strada che altri continueranno e il loro sacrificio cristologico è servito per preparare un mondo migliore. Così conclude con coerente metateatralità Edipus-scarrozzante:

Inlùdeti no d'aver vinciuto, Unifigazzione porca e'sassina! La scala è longhissema, ma là, in la cima, ce stiamo noi, no te; noi, quelli che han da perdere e crepare perchè ce sia sempre quarcheduno che poda vencerti e destruggerti! E,'desso, sarà sù el sipario, spireto del teatro. La tragedia è fenida; fenida è la triloghia; et anca la ditta dei dittanti... (p. 1374)

Simili al regno di Laio per violenza e prevaricazione sono i regni istituiti dal Totem re di Post-Hamlet ${ }^{11}$ e da Smepì nello Sfaust ${ }^{12}$, ma stavolta a preoccupare maggiormente sono i risvolti più tragici dello sviluppo capitalistico (che Testori ha visto diffondersi velocemente nell'Italia di quegli anni) : l'omologazione livellatrice, la reificazione e la progressiva disumanizzazione tesa alla riduzione dell'uomo a macchina. Le nuovissime divinità a cui si dedicano altari sono rispettivamente la Ragione ${ }^{13}$ e la Scienza che contamina tutto, tanto da attribuire la sua iniziale non solo al nome del nome del protagonista, suo adepto, ma a tanta parte dei vocaboli usati nel dramma.

Il modo di essere diversi e rifiutare un coinvolgimento annichilente con le nuove divinità da parte degli «eroi » protagonisti è però radicalmente cambiato rispetto alla Trilogia nella nuova prospettiva fideisticamente pacificata dell'autore.

La figura di Cristo vagamente allusa nel sacrificio di Edipus si concretizza nel nuovo Amleto, non più l'Ambleto paralizzato e infine soffocato da un pessimistico cupio dissolvi, ma il redentore mandato dal Padre per liberare l'umanità (rappresentata dal coro), schiava di una civiltà tecnocra- 
tica e artificiale che ha cancellato sentimenti, natura, libertà, per rimpiazzarli con "boschi di fabbriche ", "foreste di gas e di cementi ", "plastiche angosce» foriere di morte. L'irriducibilità del sacrificio di Amleto che nel duro confronto con il Totem-re, sacerdote della Ragione, arriva a perdere la lingua pur di non sottostare al siero che annulla la volontà e non perdere la sua essenza di uomo, dona al coro con il suo exemplum la forza di reagire e riacquistare attraverso il rito della comunione il senso della dignità dell'uomo, della libera espressione di sé, del rapporto con l'altro e con un corpo fatto di carne. Anche la stessa Gertrude, ennesima figura femminile che è stata fagocitata dal principio maschile del Totem-re tradendo la sua missione materna di amore, trova il coraggio di fare ammenda e unirsi al coro nel lungo cammino di rinascita.

Più tormentato è il cammino di Sfaust-scarrozzante per emanciparsi dall'impero della plastica dell' " Acna-Vernò-Vesuviana" che egli stesso inizialmente aveva contribuito a fondare e reggere siglando, nel giorno della sua incoronazione, il patto col diavolo Smepì. Fuori dai programmi di quest'ultimo fa però la sua comparsa in questo mondo pieno di fantocci di plastica senza carne e sentimenti, la procace e sensuale Margherita (o che dir si voglia "Catarina de lecch») che si mette a corteggiare il «bel Faustin» trasportandolo di botto in un Eden fatto di sensi e carnalità.

La vendetta di Smepì per il tradimento non tarda ad arrivare e nel quarto quadro compare Sfaust dilaniato dal dolore davanti al cadavere di Caterina. Il risveglio della vita, dei sensi e dell'amore è ormai avvenuto e niente può il rancoroso diavolo per ripristinare lo status quo ante; anzi, sarà proprio questo residuo di umanità che si oppone alla «Macchina» a meritargli il perdono e la salvezza ad opera di Cristo, sopraggiunto nel frattempo, a redimere l'amante prostrato.

L'amore inteso come caritas cristiana sembra dunque a Testori l'unica risposta valida in questo momento alle preoccupanti dinamiche del neocapitalismo e alla "mutazione antropologica» di pasoliniana memoria, alla spersonalizzazione dell'individuo in nome dell'artificiale e alla incipiente globalizzazione con la sua contropartita: l'emarginazione e l'isolamento dell'uomo.

L'estremo omaggio di Testori alla carità ${ }^{14}$ salvifica, sorta di testamento spirituale, può essere ritenuto il dramma sdisOrè ${ }^{15}$, omaggio che acquista ancora maggior valore dalla diversità profonda tra la riproposizione e il modello offerto dalla tradizione tragica; l'eroe portatore della carità che salverà il mondo ha qui tanta più efficacia di esempio in quanto oltre che staccarsi dalla massa corrotta e degradata dell'umanità in modo da vederne il marcio e cercare rimedio, si distacca in modo provocatorio da 
secoli di tradizione, tanto da rinunciare al suo nome e alla sua identità mitica. È quello che accade allo scarrozzante-Oreste alla fine del suo monologo drammatico: il filo della vendetta, dopo essere stato teso nel corso del dramma con l'aiuto dell'enfasi sulla negatività della figura di Clitennestra, si spezza improvvisamente mediante l'affidamento proprio ad Oreste, l'eroe della vendetta per eccellenza così come è stato consacrato teatralmente da Eschilo, un messaggio di misericordia. Oreste, una volta compiuto il duplice assassinio, si pente rinnegando il «gran macello della vendetta» in nome di concetti del tutto nuovi che non appartengono al «grechico vocabular»: il perdono e la carità. Il capo della città di Argo, nonché della chiesa (secondo il noto inscindibile binomio), chiede ad Oreste anche in nome del "traghico Estituto" di andare in esilio lontano da Argo e di rinunciare, insieme alla sua terra e ai suoi diritti, anche al nome che tale Istituto gli aveva sino ad ora assegnato. sdisOrè rinuncia dunque alla sua tradizionale identità e nel momento di congedarsi dalla città e dalla tragedia, ormai braccato dalle Erinni, fa sentire il suo grido di rivolta:

\author{
Meglio le Furie, \\ e notte, \\ e die, \\ meglio le strie, \\ ch'el vostro \\ chivile patteggiar, \\ la vostra chivile, \\ inesistenta pase! ${ }^{16}$
}

Forse però quello di sdisOrè è un viaggio verso una nuova, più vera identità. La "granda et sacra vela" di Oreste prende il largo nel tramonto e si sente nell'aria l'attesa della comparsa del Cristo- «remador » che sappia condurre l'umanità ad un diverso approdo.

Contro l'omologazione imperante va anche l'idioletto testoriano nel suo espressionismo: occorreva una lingua deformata, carnale e sanguigna per reggere all'urto del «tecnocratese» e del «televisese », nuovi linguaggi della cultura di massa; occorreva creare un precipitato di esperienze disparate in cui non piccola parte avessero i misteri e l'autenticità delle valli tanto conosciute e studiate dall'autore attraverso i pittori che le hanno ritratte ${ }^{17}$ nella loro diversità ancora non contaminata.

All'omologazione sembra però non riuscire a sottrarsi la produzione testoriana di quest'ultimo periodo, che, nel tentativo di rapportarsi ad alcuni fondamentali quesiti esistenziali in prospettiva " fideistica", finisce per diventare declinazione con poche varianti di questi. 


\section{Notes}

1. G. Testori, L'Ambleto, Milano, Rizzoli, 1972; Macbetto, Milano, Rizzoli, 1974; Edipus, Milano, Rizzoli, 1977. Prime rappresentazioni rispettivamente: 16 gennaio 1973; 21 ottobre 1974; 25 maggio 1977. La Trilogia va in scena a Milano, Salone Pier Lombardo, a cura della Cooperativa Franco Parenti. Regia di Andrée Ruth Shammah, scene e costumi di Gian Maurizio Fercioni, musiche di Fiorenzo Carpi. Interprete principale: Franco Parenti.

2. Sull'effetto generale di "diversità " suscitato da una rappresentazione romana del 1974 de L'Ambleto di Testori riflette G. Taffon nell'introduzione al suo saggio Lo scrivano, gli scarrozzanti, i templi. Giovanni Testori e il teatro, Roma, Bulzoni, 1997.

3. Così Testori stesso descrive la bizzarra sede teatrale scovata fortunosamente dalla compagnia pochi mesi prima della rappresentazione: "Non era un teatro, era un locale nato come cinema, poi trasformato in autorimessa, e poi tornato cinema, ma di quei cinema di periferia, un po'schifosetti... In mancanza di meglio, lo prendemmo, e quello divenne il salone Pier Lombardo. Il teatro era tutto squinternato. Fino a poche ore dalla 'prima'non avevamo ancora in mano il permesso di agibilità del teatro.[...] Alla fine, comunque, il permesso ci fu accordato, e il sipario potè aprirsi ». (Vedi L. Doninelli, Conversazioni con Testori, Parma, Guanda, 1993, p. 63-64. Vedi inoltre A. Bisicchia, Teatro a Milano 1968-1978. Il "Pier Lombardo" e altri spazi alternativi, Milano, Mursia, 1979.

4. G. Testori, Opere, 1965-1977, a c. di F. Panzeri, Milano, Bompiani, 1997, p. 1532. D’ora in poi, rimandiamo direttamente a questa edizione.

5. È forse sufficiente sottolineare come la tormentata omosessualità di Testori lo mettesse in condizione di toccare con mano i problemi di una sessualità diversa che si confronta con una dimensione sociale; sarebbe invece errato insistere su elementi «biografistici » per la valutazione del fatto letterario, a meno che certe costanti biografiche legate alla dimensione psicologica non siano prepotentemente entrate nel processo della scrittura (Cfr. a tal proposito F. Orlando, Dodici regole per la costruzione di un paradigma testuale, in Per una teoria freudiana della letteratura, Torino, Einaudi, 1992). La presenza dell'omosessualità si fa comunque sentire fortemente in tutta la trilogia: si pensi ai personaggi di Laerte oltre che del Franzese e di Ambleto nel primo dramma, Banco nel secondo e l'ultimo dei tre condannati dal regime di Laio nell'Edipus.

6. Quello della diversità che genera scandalo è un motivo che accomuna il macrotesto testoriano a quello pasoliniano. Il tentativo utopico di una rivoluzione contro il potere costituito e l'omologazione capitalistica, destinata però ad approdare ad una nichilistica sconfitta, avvicina in modo significativo Ambleto al personaggio di Pilade, protagonista dell'omonimo dramma di Pasolini.

7. Di nuovo un significativo punto di contatto fra Pasolini e Testori, stavolta rinvenibile nella questione del rapporto padri-figli e del conflitto generazionale; la meditazione circa la contestazione studentesca e i movimenti giovanili degli anni Sessanta-Settanta si è andata a sovrapporre ad un momento forte del vissuto di questi autori, fino a generare un nucleo tematico ricorrente nelle rispettive opere. Se Testori ha concentrato questa riflessione soprattutto all'interno della Trilogia, Pasolini l'ha invece inserita in ogni ambito della sua poliedrica attività creativa: si pensi sul versante del cinema a Uccellacci e Uccellini (1965), Edipo re (1967), Teorema (1968); al poemetto Poeta delle ceneri (1966) o al componimento Il Pci ai giovani!! (1968), alla poesia Il Coccodrillo (1968) o alle pagine introduttive delle polemiche Lettere Luterane (1976), al dramma Affabulazione (1966).

8. Eschilo, Agamennone, Milano, Rizzoli, 1995, v. 11.

9. A. Cascetta, Invito alla lettura di Giovanni Testori, Milano, Mursia, 1983, p. 110.

10. In questa declinazione didattica del triangolo freudiano "ciò che soprattutto viene estremizzato è la scelta a favore del figlio, di modo che la pulsione parricida e incestuosa non ha nessuna remora interna, ma [...] si afferma solarmente come idea di un'umanità libera e nuova ». Vedi G. Paduano, Lunga storia di Edipo re. Freud, Sofocle e il teatro occidentale, Torino, Einaudi, 1994.

11. G. Testori, Post-Hamlet, Milano, Rizzoli, 1983. Si tratta di un dramma in versi in quattro parti rappresentato per la prima volta a Milano, Teatro di Porta Romana il 12 aprile 1983 per la regia di Emanuele Banterle, scene di Gianmaurizio Fercioni, musiche di Fiorenzo Carpi. Interpreti: Adriana Innocenti, Andrea Soffiantini, Lino Troisi. 
12. G. Testori, Sfaust, Milano, Longanesi, 1990. Si tratta di un monologo in quattro quadri (dal terzo quadro in versi) che costituisce il primo dramma della "Branciatrilogia seconda ", rappresentato la prima volta a Milano al Teatro Nazionale il 22 maggio 1990 per la regia di Giovanni Testori. Interprete: Franco Branciaroli.

13. Di nuovo una consonanza col Pilade di Pasolini, che prospetta in forma drammatica alcune delle riflessioni che Pasolini già alla fine degli anni Sessanta andava disseminando nelle colonne dei quotidiani italiani : l'improvviso ed enorme salto fra « una cultura fatta di analfabetismo (il popolo) e di umanesimo cencioso (i ceti medi) » caratterizzata da "un'organizzazione culturale arcaica» e " l'organizzazione moderna della cultura di massa ", da Pasolini definito "mutazione antropologica ", rischia di spazzare via l' " universo agricolo paleocapitalistico » con la sua cultura, il suo dialetto, i suoi culti arcaici e ciclici alcora legati al paganesimo. Tale mutazione è quella imposta violentemente da Oreste nella "primitiva» città di Argo grazie alla guida e alla volontà di Atena, dea della Ragione e del nuovo sviluppo capitalistico imposto alla città.

14. Nello stesso anno di composizione dello sdisOrè l'autore stava lavorando alla traduzione della prima lettera ai Corinzi di san Paolo (G. Testori, Traduzione della prima lettera ai Corinti, Milano, Longanesi, 1991) il cui messaggio fondamentale, basato appunto sulla carità portatrice di perdono e riconciliazione, viene tradotto in forma drammatica nella vicenda di Oreste.

15. G. Testori, sdisOrè, Milano, Longanesi, 1991.

16. Ibid., p. 118.

17. Il riferimento è a Giacomo Ceruti, Giovan Battista Moroni, Gaudenzio Ferrari, il Romanino ed altri pittori ben conosciuti e studiati dall'autore durante la sua lunga attività di critico d'arte. Gli scritti di Testori intorno a questi autori sono stati raccolti nel volume La realtà della pittura. Scritti di storia e critica d'arte dal Quattrocento al Settecento, a c. di P. C. Marani, Milano, Longanesi, 1995. 